\section{The Rat Brain in Stereotaxic Coordinates (2nd edn)}

by George Paxinos and Charles Watson, Academic Press, 1986. $\mathrm{E40.00/}$ $\$ 80.00$ (264 pages) ISBN 012547 6213

The second editon of Paxinos and Watson's rat stereotaxic atlas is, like the first, a detailed and definitive resource for stereotaxic surgery, brain dissection and neuroanatomical instruction. It offers accurate and detailed stereotaxic coordinates in three planes of section (coronal, horizontal and sagittal), and is presented in a clear, coherent manner. The photomicrographs included are well-reproduced and informative and the corresponding linedrawings detailed and thoroughly annotated. The nomenclature employed is clear and useful. As such, it is, like the first edition, an indispensable commodity for neuroscientists utilizing rodent models for research as well as for students learning basic fundamentals of rat brain neuroanatomy.
However, the second edition is far more than a mere recapitulation or update of the first. Most notably, the authors have approximately doubled the number of plates and line drawings, yielding a greater sampling density through the brain (roughly every $0.25 \mathrm{~mm}$ in coronal and horizontal sections, as opposed to about 0.40 to 0.50 $\mathrm{mm}$ in the first edition). It is clear from the added plates and drawings that changes in brain nuclear and subnuclear structure can be quite dramatic within $0.50 \mathrm{~mm}$ and that the increase in illustrations were sorely needed; indeed, sampling density was one of the few criticisms of the first edition. The authors have also included a more detailed analysis of the cytoarchitecture of brain structures, a product of both extensive consultation with numerous authorities on neuroanatomical organization of brain nuclei and careful analysis of their own material. The criteria used to make these changes were wellannotated in the introduction to the second edition, and were based on cytoarchitectonic distinctions and, perhaps more importantly, on distinctions derived from contemporary histochemical techniques. The latter improvement clearly represents a successful attempt on the authors' part to incorporate information derived from modern chemical neuroanatomy in determining their coordinate system, and represents one strong point of this second edition.

In sum, the second edition of Paxinos and Watson is every bit as indispensable to the neuroscientist and neuroscience student as was the first. In preparing this second edition, the authors have answered some of the limited criticisms of the first (for example, see Swanson's comments in the February 1984 issue of TINS and have taken into consideration state-of-the-art neuroanatomy. The Rat Brain in Stereotaxic Coordinates (2nd edn) stands alone as a research and instructional resource for the neuroscientist of the 1980s.

\section{Blindsight: A Case Study and Implications}

by L. Weiskrantz, Oxford University Press, 1986. 119.50 (viii +187 pages) ISBN 9188521294

Ever since Gordon Holmes described a consistent topographic relationship between visual field defects and wounds of the occipital lobe, geniculostriate processing has been considered indispensable to vision. In this book, Professor Larry Weiskrantz advances a challenging hypothesis that predicts residual vision despite striate cortex lesions. In Part I of his monograph, he theorizes that non-geniculostriate projections through the diencephalon do more than merely serve reflex functions such as eye blink. This substantial network may possess additional properties that permit useful visual discriminations even without our awareness.

The original impetus for 'blind- sight' research followed experimental observations in animals. After the complete removal of visual cortex, monkeys are not rendered blind. Although they can no longer recognize other monkeys or discriminate patterns and colors, they can still reach for peripheral objects and avoid obstacles better than by sheer chance. Assuming that the ablations were complete (this has been disputed), the only way to explain the remaining ability is to postulate the function of visual areas untouched by the experiments. The best candidate for the role is the phylogenetically older 'second' visual system, previously delegated to a vestigial role in primates. This system has major subcortical relays from the retina to the pulvinar and superior colliculus, whence it projects secondarily to prestriate visual cortex. Layer IV of Brodmann's area 17. the recipient of geniculostriate impulses, is avoided altogether. Do humans also have such an alternative visual pathway, or does the finding in monkey reflect the greater importance of subcortical pathways in less advanced species?

'Blindsight' is the selfcontradictory term that has come to describe the residual visual behavior in humans that is comparable to the findings in primates. Aided by nature, neurosurgery, and a familiarity with the work of Ernst Poppel, Weiskrantz and his colleague Elizabeth Warrington sought to test the blindsight hypothesis. Since 1974 they have collaborated in the experimental study of human subject D. B., who had a portion of his visual cortex removed during surgery for the removal of a vascular malformation. 'From the surgical notes it was estimated that the incision extended from the occipital pole forward by approximately $6 \mathrm{~cm}$ and was thought to include a major portion of the calcarine cortex - in which the striate cortex is situated - in the right hemisphere.'

Immediately after surgery,
James P. Herman and Stanley J. Watson Mental Health Research Institute, University of Michigan, Ann Arbor, MI 48109, USA

\section{Matthew Rizzo \\ Division of Behavioral Neurology, Department of Neurology, The University of lowa College of Medicine, lowa City, IA 52242 USA}

